\title{
Catching the Wave
}

\author{
Amnon Yariv, Life Fellow, IEEE
}

Invited Paper

\section{Index Terms-Integrated optics, semiconductor lasers.}

\section{ISRAEL TO BERKELEY}

$\mathbf{I}$ N THE summer of 1950, I was fresh out of the Israeli Army where I had served in (the first) artillery unit during the war of independence. I saw military action on the Jordanian-Jerusalem and the Egyptian fronts, and then served for a year as an instructor in the Artillery Officers School. (My first publication is a photo manual on the use of a German $50 \mathrm{~mm}$ gun.) After my discharge in 1950, I needed to decide on my next step in life.

The decision was not easy since I had no single burning passion. I had attended a rigorous scientific program at Geula High School in my home town of Tel Aviv, where my favorite subjects were languages, which in addition to Hebrew consisted of English, French, and Arabic. I enjoyed sports: basketball and rowing in high school and mostly body surfing. In the fall and winter, the Mediterranean could kick up some decent surf. (Hebrew at that time had no word for surfing - probably because it was not practiced in biblical times - so we used the term "to catch a wave" and referred to our group as the "Wavecatchers Association.") This was my first introduction to waves and to a lifelong activity. Back to my dilemma: without a clear cut preference of what to do-except a strong conviction that I was not ready to join the world's proletariat as yet, I decided to go to college. With my mathematical-scientific high school diploma, I applied to The Technion-Israeli Institute of Technology in Haifa and was accepted to study electrical engineering starting in September of 1950.

I spent the intervening months working on a surveying crew for the ministry of agriculture, getting to know first hand (or rather first foot) the new Israel. A chance encounter on a bus in Tel Aviv some time in August of that year was to change radically my future. In the bus I met Ben, an army buddy who was on his way to the U.S. consulate to apply for a student Visa. Ben told me that San Mateo Junior College in California just "loved" Israeli students and exempted them from tuition. The thought of studying abroad had not occurred to me up to that time. In the following week, I dashed application forms to San Mateo Junior College and, as an insurance, to Columbia University and UC Berkeley. To my great surprise, I received accep-

Manuscript received October 31, 2000.

The author is with California Institute of Technology, Pasadena, CA 91125-0001 USA.

Publisher Item Identifier S 1077-260X(00)11633-8. tances to all three schools within two months, and on December 31, 1950, found myself on a freighter bound for Baltimore. I had decided to attend San Mateo Junior College where I would room with Ben. The main reason for choosing the school was its no-tuition policy. A second and strong reason was that as a teenager I was greatly attracted to the books of Jack London (in translation) and his evocative descriptions of Northern California. An unintended result of my choice of school was the fact that the United States I found upon arrival was the beautiful San Francisco peninsula with its ocean and bay vistas and redwood forests. It took me a while to discover that towns such as Hillsborough, Palo Alto, and Atherton were not typical of the rest of the country.

San Mateo Junior College was a pleasant introduction to the United States. It was rigorous and most of its graduates went on to Berkeley. In my case, I decided to move to UC Berkeley after one term. A waiver of my nonresident tuition fee was a big help. After a summer spent working in a Hayward cannery on the east San Francisco bay driving a forklift, a job I would keep for the next three summers and that required that I join the AFL-CIO, I transferred to Berkeley as a sophomore in Electrical Engineering.

Berkeley in 1951 was already an active and cosmopolitan campus. The variety of things to do on campus and the proximity to exotic San Francisco across the bay made it a wonderful college experience. Odd jobs as a painter, driver, and gardener, plus the cannery work during the summers, helped pay the bills, and I picked "cheap" dates by ushering together at the opera house and the Geary theater. At the beginning I found myself mostly in large classes taught by teaching assistants. By the end of the second year, however, I started taking smaller classes with some of the great professors. My emphasis up to that point was on control and machinery. An elective course in Electromagnetic (EM) Waves taught by Professor John Whinnery was a major turning point. I found it more interesting and more enjoyable than any course I had taken up to that point and it struck a sympathetic resonance in me. Maybe related to my earlier "Wavecatchers Association" days. The result was that after graduation in 1954 I decided to join Whinnery's research group as a new graduate student. This decision was made easier by the award of a \$2000/year fellowship by IBM. I used part of the money to go to Israel and marry Jeanne, whom I had met and dated in Berkeley.

Life as a graduate student in Berkeley during the late 1950's was pleasant. The student riots were still a decade off and the IBM fellowship removed most of the financial worries. The main research topic in Whinnery's group was the traveling wave 
tube (TWT). TWTs were used as microwave amplifiers and oscillators. In retrospect, this was an excellent, almost tailormade, introduction for someone who would later pursue a career in waves - any waves. The TWT amplifies an electromagnetic wave propagating in a periodic waveguide (usually a helix) by shooting an electron beam in close proximity to the EM wave. One of the best ways to understand the physics of the amplification process is to use the coupled-mode point of view which had first been introduced by John Pierce [1]. Using Pierce's point of view, power is exchanged between space charge waves (waves of velocity and density modulation) on the electron beam and the EM mode. This was my first introduction to the coupled-mode point of view (which I would apply and reapply in my subsequent research) and was the topic of my first paper [2]. An immediate and key consequence of the coupled-mode theory was that a prerequisite for power exchange between interacting modes was a near synchronism of the velocities of the interacting modes. This concept came to be known, much later, in the Nonlinear Optics community as "phase matching." The otherwise impossible velocity matching between a "slow" electron beam and an EM wave was made possible by the fact that the EM guide was periodic. That meant that the EM mode consisted not of a simple fast wave but of a multiplicity of wavelets (spatial harmonics) propagating, each with a phase velocity

$$
v_{m}=\frac{\omega}{\beta_{0} \pm m \frac{2 \pi}{\Lambda}}, \quad m= \pm 1,2,3, \cdots
$$

and (all) oscillating at the same radian frequency $\omega . \beta_{0}$ is the propagation constant of a straight uniform waveguide, $m$ is the mode index, and $\Lambda$ is the spatial period of the EM guide. In order to obtain interaction between the beam and the EM mode, one could choose an integer $m$ such that synchronism, $v_{m} \approx$ $v_{\text {electron, was achieved. Condition (1) is identical to the principle }}$ of quasi-phase matching in nonlinear optics [3]. In addition, it was necessary to solve the electromagnetic mode propagation problem in the waveguide. The fact that I was accumulating a treasure trove of basic concepts was not obvious to me at the time, but it probably subconsciously influenced my future research. After writing a master's thesis on TWT interactions, I started looking for a Ph.D. project. John Whinnery and his gracious wife Patricia had become surrogate parents to all of us in the group, and the thought of leaving Berkeley to pursue my Ph.D. degree at another school never entered my mind. I decided, however, to seek a new, non TWT, topic for my doctoral research.

In 1956, I went to Boulder, CO, to attend the highly informal annual "tube" conference, an event attended by the friends and colleagues of Whinnery, John Pierce, Rudy Kompfner, Cal Quate, Chapin Culter, and other kindred spirits, mostly from the Bell Telephone Laboratories. This was the venue for much of the recent research results in communication related technologies. In that meeting, I heard George Feher of Bell Labs talk about a two-level maser amplifier, the microwave precursor to the laser. I did not understand most of the underlying physics, especially the quantum mechanics of the electron spin in a magnetic field, but I fell under the spell of the topic. I decided to choose this new area as my research topic. John Whinnery agreed to continue to serve as my thesis professor in spite of the fact that he too knew little of the requisite physics.

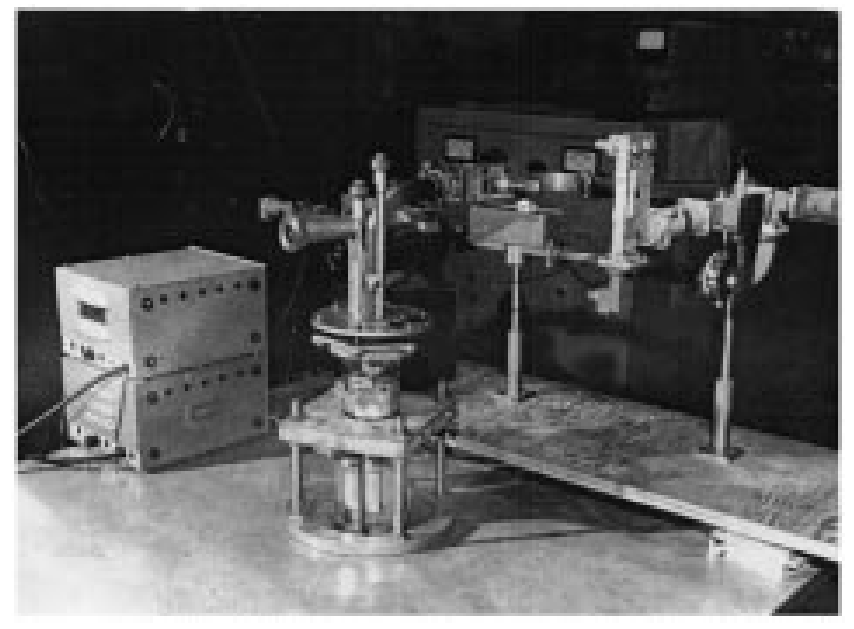

Fig. 1. My experimental setup for observing maser action in inverted spins in a magnetic field (magnet below surface not shown), U.C. Berkeley, 1958.

The first step was to enroll in a whole slew of physics courses. This was probably my hardest year in Berkeley. During that year, I took courses in basic quantum mechanics (Emilio Segre), advanced quantum mechanics (Nierenberg), paramagnetic resonance (Hahn), nuclear physics (Alvarez), and group theory (Tinkham). I also managed to spend a couple of weeks with George Feher at Bell Labs (George too had come from Israel and had studied physics in Berkeley) and learned how his microwave electron paramagnetic system worked. At the same time, I started setting up my own experimental setup (see Fig. 1). The latter consisted of a reflection-type microwave cavity placed in a liquid He dewar in a magnetic field and fed by a pound-stabilized microwave oscillator. My experiment consisted of inverting the spin populations of a two-level system by adiabatic fast passage and then watch the periodic and coherent exchange of energy between the spin-system and the microwave cavity. The theoretical background was the classical work of Bloembergen Purcell and Pound in nuclear resonance [4], [36]. I succeeded in observing maser-type amplification of an incident microwave signal and started writing up my thesis. I believe that mine was the first quantum electronics research at UC Berkeley. Of course, at Columbia University, C. H. Townes and his students J. P. Gordon and H. Zeiger were already deeply involved in the Ammonia Maser.

John Whinnery took my word for it when I told him that I had already done enough for a Ph.D. thesis. I defended my thesis in October 1958 and was ready to go. Our group had been visited frequently by some of John Whinnery's colleagues from Bell Labs. This group included some extraordinary people whose names I already mentioned such as John Pierce, Chapin Cutler, the Austrian-Jewish architect turned electrical engineer Rudy Kompfner, Calvin Quate, and others. In a way, I was already recruited to Bell Labs before even seeing it on the basis of its emissaries to Berkeley.

\section{BELL LABORATORIES}

I landed in Newark, NJ, in January 1959 with pregnant Jeanne during a blistering cold spell. It would be April before I discovered that New Jersey is actually beautiful (that is, if you ignore the part of the state near the Hudson river and the New Jersey 
turnpike). The problem I encountered at Bell Laboratories (Bell) was that nobody told me what to do. How could I be sure that the topic I would choose would be up to the standards of this world-renowned institution? Because of my microwave background, I got involved in taking advantage of the negative resistance of the newly discovered Esaki Tunnel Diode to make a microwave amplifier. In a way, it was like my Ph.D. experiment-put a "negative resistor" inside a microwave resonator which terminates a waveguide and the reflected signal can exceed that of the input, i.e., gain. I got my first pat on the back when my project was chosen for display at that year's show at the National Academy of Sciences. I also did some paramagnetic resonance work and was involved in the search and race for the first optical maser (laser). There were three groups at Bell at the time pursuing the holy grail of a laser. A group at the Electronics Research department with Gary Boyd and myself working on uranium-doped $\mathrm{CaF}_{2}$ a group consisting of Bennet, Herriott, and Ali Javan working on $\mathrm{He}-\mathrm{Ne}$, and another group working on rare earth ions in $\mathrm{CaWO}_{4}$. The practically unlimited resources of Bell Labs were very much at play. I had about six to seven engineers and technicians help me cut, polish, orient, and test a large range of potential laser crystals. It was only later that somebody would explain to me the reason for this unbounded largesse. As a regulated monopoly, research at the A.T.T. Bell Laboratories was an allowed expense. Something like the buying of telephone poles. So not only was research free-since A.T.T. was allowed a fixed return on its allowed expenses - the company actually made a profit on the research. (If one is tempted to denigrate this system, I would remind him that it was under this system that the transistor and many other key technologies were invented.) While the three groups at Bell were racing each other to make the world's first laser, unbeknownst to us, Theodore Maiman at the Hughes Research Laboratories at Malibu, California, was pursuing his own agenda. Working alone and with minimal encouragement, he made the world's first laser in July 1960 using a flashpumped ruby crystal $\left(\mathrm{Al}_{2} \mathrm{O}_{3}\right.$ doped with $\mathrm{Cr}^{3+}$ ). I received the news while on vacation in San Diego with Jeanne and baby daughter Danielle. Jim Gordon, my boss at Bell, called me and asked that I take a day off and drive up the coast to Malibu to check the story out.

Imagine yourself in the position of Ted Maiman claiming the world's first laser. Afraid that he might be ridiculed if he were wrong, he nervously and readily showed me the data-a sudden collapse, above threshold, of the fluorescence spot diameter to a diffraction-limited spot and the corresponding sudden increase of the peak brightness. I called Jim back that evening to tell him that, in my opinion, Ted had made the world's first laser.

The groups at Bell continued their frantic search and eventually wound up with a number of new lasers including the first gas laser (He-Ne) of Javan's group. But it was Maiman, working alone, who won the race. In the years which followed, I often thought about Maiman and his work. At Bell Labs, we were all trying to make CW lasers. The thought of pulse excitation of a laser-as Maiman had done-had not occurred to us. We were probably influencing each other to the exclusion of different approaches. I would often tell this story to my classes at Caltech as a warning against a herd psychology in research.

I believe that Maiman didn't receive the credit he deserved partly because he left the research world soon after his demonstration of the laser, and partly because he did not have the po- litical backing of a major organization which, unfortunately, is a prerequisite for major awards.

Back to Bell. In the summer of 1960, I attended the first international conference in quantum electronics at Shawanga Lodge in the Catskill mountains. Most of the big wigs in the field were there. I believe that the 100 or so attendees included most of the world's laser researchers. Among the papers given was one by $\mathrm{H}$. Heffner, a creative electrical engineering professor from Stanford, on parametric amplifiers (PAs). This new type of microwave amplifier transferred power from a "pump" field at a frequency $\omega_{p}$ to, simultaneously, a "signal" and "idler" fields at $\omega_{s}$ and $\omega_{i}$, respectively, where

$$
\omega_{p}=\omega_{i}+\omega_{s}
$$

The physical principle involved was a modulation by the "pump" field of a reactive component, a capacitance, or an inductance. Heffner claimed in his talk that the PA was a noiseless amplifier, i.e., that it could amplify a signal without adding noise so that the signal-to-noise power ratio after amplification remained the same. This would make the PA a better amplifier than the laser. Shimoda, Takahaski, and Townes had previously shown in 1957 that a maser amplified signal obeyed the uncertainty principle [5]

$$
\Delta n \Delta \phi=\frac{1}{2}
$$

for the fluctuations $\Delta n$ and $\Delta \phi$ in its number of quanta and phase, respectively. An uncertainty $\Delta n$ is what we would call today amplitude noise (or AM noise) while $\Delta \phi$ is frequency (or phase) FM noise.

In a comment included in the conference proceedings, I suggested that the comparison of the PA to a laser amplifier could not be based on a classical analysis such as Heffner's, since the prediction of the laser noise resulted from a quantum mechanical analysis. Thus, what was needed was a corresponding quantum analysis of the parametric amplifier. Upon return to Bell, this became my principal occupation. It required learning some quantum field theory and starting a close collaboration with Bill Louisell—a talented mathematical physicist. The key result was to explain the interaction between the pump and signal and idler fields in terms of a nonlinear Hamiltonian

$$
H_{\text {interaction }}=-\hbar \kappa\left[a_{s}^{\dagger} a_{i}^{\dagger} e^{-i\left(\omega_{p} t+\phi\right)}+a_{s} a_{i} e^{i\left(\omega_{p} t+\phi\right)}\right]
$$

where the $a^{\dagger}$ 's are the "creation" operators and the $a$ 's the annihilation operators for the respective signal and idler fields and $\omega_{p}=\omega_{i}+\omega_{s}$. With the interaction Hamiltonian and the exceedingly elegant properties of the $a^{\dagger}$ and $a$ operators, it was only one short step to show that the parametric amplifier obeyed the same uncertainty relations (3) as the laser. A byproduct, unexpected, of the analysis was the prediction of spontaneous parametric fluorescence. This was gratifying. The process also left me a strong convert to the power of quantum mechanics. The paper [6] describing the work is, arguably, the first paper in nonlinear quantum optics and the Hamiltonian (4) is the starting point for nearly all work in the field. It is also used in the analysis and prediction of optical squeezing [7].

One of the most exciting aspects of the work at Bell was the association with talented colleagues, most of them new recruits like myself. Our small department included J. P. Gordon, who, as Townes' student at Columbia, made the first ammonia laser 


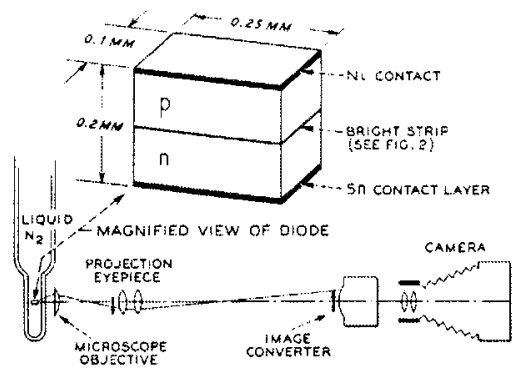

Fig. 2. Observation of dielectric waveguiding in GaAs p-n junction lasers.

and years later would make with Herman Haus major contributions to the soliton area, Kumar Patel discovering new gas lasers by the week, and Gary Boyd and Herwig Kogelnik with their now classic work on Gaussian optical beam modes. I would also interact closely with J. Giordmaine (inventor of crystal phase matching), Ivan Kaminow (microwave modulation of lasers), P. $\mathrm{K}$. Tien, and J. Geusic with his Nd: YAG laser. Especially productive in my case was the interaction with Herwig Kogelnik a gem of a (plasma) scientist who had been discovered by R. Kompfner in the wilds of Austria. Combining Kogelnik's mastery of Gaussian Modes with my background in the physics of spontaneous and induced transitions we showed that to make an ideal laser amplifier required spatial and temporal filtering. We also derived the equivalent noise input power [8] of an ideal laser amplifier

$$
P_{\text {noise.equiv. }}=h \nu \Delta \nu(G-1)
$$

( $G$ is the optical power gain, $\Delta \nu$ the optical bandwidth) of an optical signal at frequency $\nu$. This expression and the relevant discussion would become practically important many years later with the advent and technological prominence of optical fiber amplifiers.

My honeymoon at Bell came to an end one day in 1963. A few months earlier, a semiconductor laser (SCL), using a GaAs p-n junction, had been announced by groups at the IBM and General Electric research laboratories. Rogerio Leite and I were requested to take a look at this new "animal" and make sure that Bell was not missing out on something important. With the help of Barry Cohen, who knew how to make p-n junctions, and Walter Bond, a master experimentalist who could make anything work, we succeeded in replicating in a short time the GE and IBM work. This was my introduction to the world of semiconductors - an involvement that continues to this day. In trying to understand how the semiconductor laser worked, we ran into a conceptual wall. By photographing the front facet of the laser, we could determine the beam diameter (see Fig. 2) and found it to be approximate $1 \mu \mathrm{m}$. Such a narrow beam would diffract in its return propagation to a spot of $300 \mu \mathrm{m}$ which would constitute an immense loss. A loss far greater than that which could be compensated by the available gain in the narrow active region (2-3 $\mu \mathrm{m})$ straddling the $\mathrm{p}-\mathrm{n}$ junction. The only way to reconcile these seemingly contradicting inputs was to postulate that a waveguiding mechanism confined the laser beam essentially to a short transverse distance near the p-n junction where the gain due to the simultaneous presence of electrons and holes

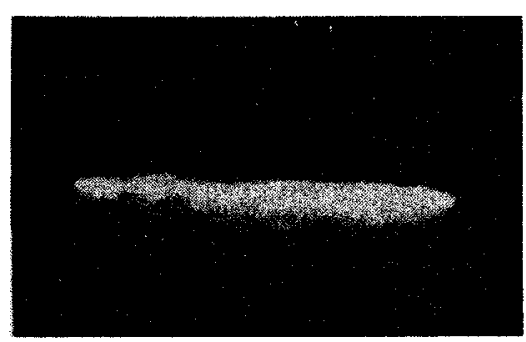

was concentrated. In other words: waveguiding was a prerequisite for lasing in SC lasers [9], [37]. The mechanism we proposed as responsible for waveguiding was a reduction of the dielectric constant (or index of refraction) in the heavily doped $\mathrm{p}$ and $\mathrm{n}$ regions adjacent to the junction due to the presence of mobile charge carriers: the plasma effect. In retrospect, it is interesting that although our work at Bell was the first to point out the crucial role of optical waveguiding in SC lasers, we did not carry the idea to the next logical step of creating a deliberate strong waveguide to enhance the effect. That would be left to the Ioffe Institute group of Alferov in Leningrad who used GaAlAs-GaAs-GaAlAs to create a waveguide straddling the pn junction. This step laid the foundation later for achieving $\mathrm{CW}$ room-temperature lasing by Hayashi, Panish, and Foy in 1969. Alferov and Herbert Kroemer, who proposed it independently, would win the 2000 Nobel prize in physics for this work.

\section{CALtech}

In 1964, I decided to leave Bell and return to California. The decision was not well reasoned or thought out. I think it had to do with wanting to return to the sunny climate and beaches of California and some notion that a university career would offer a more varied and interesting experience. In 1964, I started as an Associate Professor of electrical engineering at the California Institute of Technology (Caltech) in Pasadena. My "recruiter" to Caltech was Roy Gould, a Professor of electrical engineering and of physics whose elegant work on waves in magnetized plasmas I had studied as a student in Berkeley. My first project at Caltech was to set up a research laboratory. I decided to pursue work in semiconductor lasers since the combination of semiconductor device physics (pn junctions, etc), electromagnetics (optical guiding, resonator modes), and quantum electronics (stimulated emission from a Fermi-Dirac inverted population of carriers) seemed to offer wide ranging opportunities for research and thus for Ph.D. theses. The thought of SC lasers as the engine of optical communication networks was not on anybody's radar scope.

Between Bell and Caltech, I had spent the summer at a company, Watkins-Johnson (WJ), in Palo Alto. My second daughter Dana was born at the Stanford University Hospital in 1964. At WJ, I taught myself the theory of light propagation in crystals and the electrooptic effect since the company was interested in modulation of laser radiation. This background proved useful and was my introduction to the world of nonlinear optics. (Yes - the linear electrooptic effect is a nonlinear optical effect.) 
The interest in modulation thus led me in 1964 to read a paper by Gürs and Müller on internal modulation of lasers, i.e., modulation inside the laser resonator [10]. They observed that when the modulation frequency was equal to that of the longitudinal mode spacing $c / 2 n l$, the laser output would become unstable. In an attempt to analyze what happened, I came up with a coupled-mode picture, known today as mode locking [11]. In the presence of loss or index modulation inside the laser resonator, the independent mode solution of an ordinary laser is no longer a "good" solution since it does not satisfy Maxwell's equations with a time-dependent $\epsilon$ or $\sigma$ (loss). Instead-one has to consider a field made up of a superposition of laser modes which are equispaced in frequency. A substitution of such a superposition into Maxwell's equation results in a recurrence equation for the mode amplitudes which are now mutually locked in phase and amplitude. Viewed in the time domain, this superposition gives rise to modelocked ultrashort pulses whose time duration is limited only by the spectral width of the laser gain mechanism. On the way to Ithaca to deliver a paper on my work, I stopped at Bell Labs and gave a seminar on mode locking and ultrashort pulses only to discover that Hargrove, Fork, and Pollack had just observed this phenomenon. Both their work [12] and mine were presented in the informal Electron Device Research Conference at Cornell University in 1964. Personally, the work on mode locking was among the more enjoyable ideas I have worked on in my career and among the most dramatic manifestations of wave interference. It appealed to both the theoretician and the engineer in me. It was also a demonstration of how entirely two different points of view - that of a time gating and survival of the fittest which was used by Hargrove et al. and my formal mathematical approach of a coherent superposition of eigenmodes which was needed to satisfy Maxwell's equations-converged to the same final picture and invention, ultrashort pulses.

\section{A. Optoelectronic Integrated Circuits (OEICs)}

Back at Caltech, in the meantime, I had picked a few graduate students and was on the lookout for "good" research projects. At the absence of any great ideas, I set them to work on the only topics with which I had some experience: 1) laser mode locking and 2) optical waveguiding in semiconductors and SC lasers. The hope was that once we all got our hands dirty we would find something interesting to do (in retrospect, this recipe seems to work). Student No. 1, John Zoutendyk, did his research on InAs lasers, Jean Pierre Laussade and Jack Comly on self mode locking by Kerr media in a laser, my fourth student David Hall agreed to work on waveguiding in semiconductors (SC) - a courageous decision since our SC processing capability was minimal.

Instead of aiming for guiding in p-n junctions, as we had done at Bell, we decided to have the guiding take place in a surface waveguide which results when a high-resistivity GaAs layer is grown epitaxially on a low-resistivity substrate. The expected waveguiding in this case would be caused by a lowering of the dielectric constant in the low-resistivity substrate relative to that of the surface layer by

$$
\delta \epsilon=-\frac{N e^{2}}{m^{*} \omega^{2}}
$$

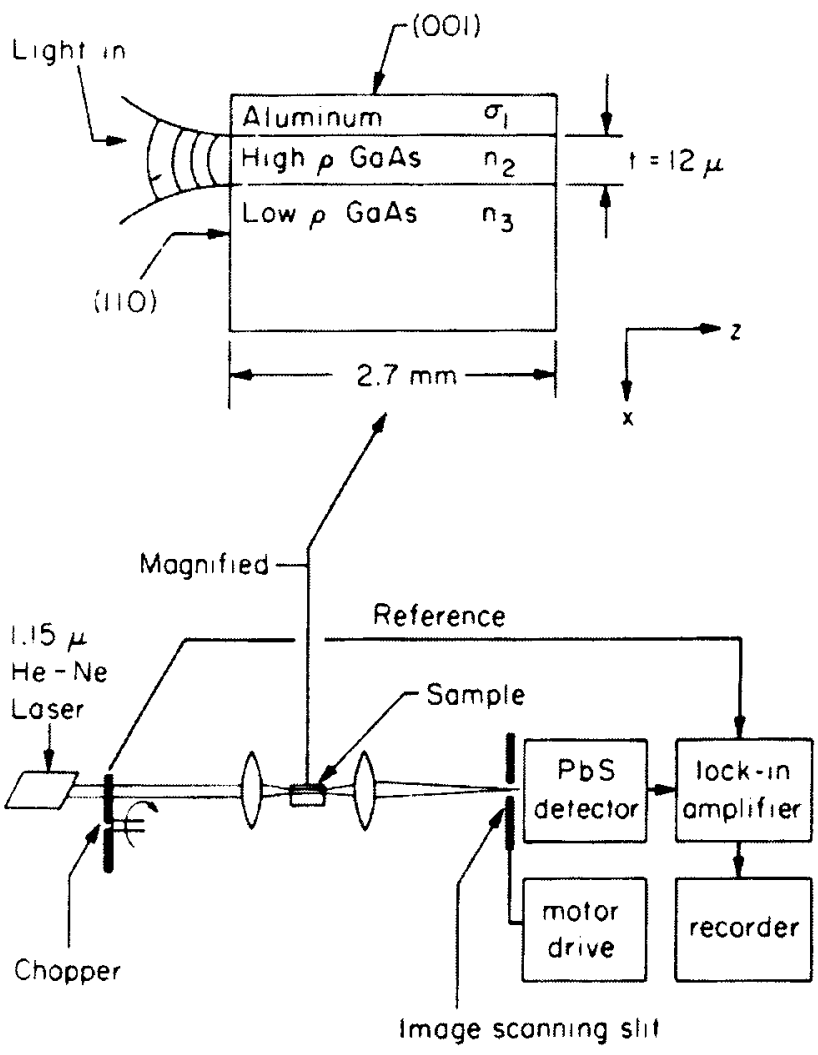

Fig. 3. David Hall's electrooptic waveguide modulator.

where $N$ is the carrier density in the high conductivity substrate and $m^{*}$ is the effective mass of the carriers. It so happened that such structures were being fabricated at the time by researchers working on the Gunn effect, and we hoped that some of the Gunn samples would fit our requirements.

David Hall spent the better part of two years looking for guiding in many crystal samples, which were donated by various laboratories, with no success. Sometime toward the spring of 1969, we agreed that, if by the end of the summer we could not observe optical guiding, David would abandon the experiment and move on to some "safe" project. Early that summer, he went home to Hartford, CT, on vacation and there discovered a new, yet untapped by us, source of crystals at the United Aircraft Research Laboratories. By the end of the summer, Dave would process the crystals into appropriate samples and almost immediately, working with Elsa Garmire, who in the meantime joined us as a Post-Doctoral fellow, observed optical waveguiding. My own excitement was exceeded only by that of Dave to whom the bright strip of light on the image converter signified not only a successful experiment, but also a good part of his Ph.D. thesis. Fig. 3 is a reproduction from the 1970 paper [13] describing the work. You will note that, in addition to the waveguide, we also had a metal electrode on top of the crystal. This small but significant addition to the experiment made it possible to control the index of refraction in the guiding region by applying a (reverse) voltage between the substrate and the metal. The resulting depletion layer (Schottky barrier) electric field acting through the electrooptic effect of GaAs changes the dielectric constant of the guiding layer by

$$
\delta \epsilon=\epsilon_{0} n^{4} r_{41} E
$$


where

$r_{41}$ electrooptic coefficient of GaAs;

$n \quad$ its index of refraction;

$E \quad$ electric field.

The total change of the dielectric constant of the guiding channel $\delta \epsilon$ is thus the sum of (6) and (7), so that, with a proper choice of the sign of $E, \delta \epsilon$ can be made to drop below the threshold value (for guiding) and the guiding would (and did) disappear. We thus had a voltage-controlled dielectric waveguide which could be used as a light modulator. This was, possibly, the first guided-wave electrooptic modulator, a technique that, based mostly on $\mathrm{LiNbO}_{3}$ as a crystal, would later become the mainstay for today's optical switching technology.

The next development in the field to impact our work was the publication in 1968 of an article by Shubert and Harris [14], then at the University of Washington, with the title of "Optical Surface Waves on Thin Films and their Applications to Integrated Data Processors." This paper was the first to suggest "... the applicability of thin films to optical data processing ... in which the thin film serves as the transmission medium ... and important elements are lenses, modulators and detectors," realized in thin film (i.e., dielectric waveguide) configuration.

Reading the Shubert and Harris paper made me, for the first time, look beyond the narrow conceptual boundaries of our guiding and modulation experiment in GaAs. It became clear that GaAs was almost unique in the sense that it could "play" optical games (guiding), perform electrooptical modulation since it had a sizable electrooptical coefficient, detect light (p-n junction), make excellent lasers, as well as act as a base material for purely electronic devices (metal-oxide field-effect transistors, Impatt, Gunn diodes, etc.). This meant that, in principle, we should be able to integrate any desired combination of these devices monolithically on a single substrate of GaAs. I described this possibility in the first Symposium devoted to this new field which was organized by R. L. Byer (Stanford) and O. Bryngdahl (XEROX, PARC) in Menlo Park, CA, in March 1971. To distinguish this field from its older brother, integrated optics, I referred to it as "Active Integrated Optics." Later that year in a meeting in Esfahan, Iran, organized by A. Javan, I came back to the same topic. I will take here the liberty of reproducing a few paragraphs from the proceedings of the 1971 conference [15] which deal with the issue of Integrated Optoelectronics Circuits.

"... just as silicon and germanium have come to play a key role in integrated electronics, it is possible already to develop approximate criteria which point toward certain materials as candidates for active integrated optics applications. Some of the more important requisite properties are:

1) transparency and good optical quality for light in the visible and near-visible regions of the spectrum.

2) material should lend itself easily to interfacing with electronic circuits.

3) the material should be capable of light generation and detection.

4) the material should be capable of performing light switching and modulation functions. More specifically, it should possess large electrooptic and photoelastic figures of merit so that modulation and switching of light by either of these two techniques can be used.

5) the material should be suitable for thin-film dielectric waveguide fabrication. There are many materials that can satisfy reasonably well one or two of these requirements and it is conceivable that future integrated circuits will combine a number of them for specific applications. It is interesting to note, however, that at least one class of known materials already comes close to fulfilling all of these requirements. This is the semiconductor GaAs and its related alloys, such as $\mathrm{Ga}_{1-x} \mathrm{Al}_{x}$ As and $\mathrm{GaAs}_{1-x} \mathrm{P}_{x}$."

In a meeting in a semiconductor conference in Japan in 1970, I described some of the ideas about integrated optoelectronics circuits. After the talk, I was approached in the conference hallway by a gentleman I had not met before. He introduced himself as Dr. Martin Stickley, a program director at Defense Advanced Projects Agency (DARPA), and offered to support a research project to realize my proposal of Integrated Optoelectronic Circuits (later to be known as OEICs-optoelectronic integrated circuits). Needless to say, I accepted this offer on the spot and have continued to work with DARPA to this date.

At that time, it became clear to us that, to make further progress in this field, we would need to be able to grow our GaAs- $\mathrm{Ga}_{x} \mathrm{Al}_{1-x}$ As structures. This, at the time, seemed to be a scary excursion into the black magic land of crystal growth. With the help of I. Samid, a Post-Doctoral fellow from Israel, and Elsa Garmire, we set up two liquid phase epitaxial reactors and, by 1972, were growing our own crystals. In retrospect, this was a crucial decision for us since the increasing complexity of the devices which were required made it all but impossible to get them from any other source but our own. It also pointed the way to the unavoidable need to develop sophisticated semiconductor growth, fabrication, and test facilities at universities in order to perform meaningful research and train students in a new and technologically demanding area.

Over the next two years, our group at Caltech and, specifically, Nadav Bar Chaim, a Post-Doctoral from Israel, Israel Ury, a graduate student, and Shlomo Margalit, another visitor from the Technion, later a co-founder of MRV, succeeded in demonstrating a number of OEICs involving monolithic integration of detectors, lasers, MESFET's, and modulators on a single semiconductor crystal substrate. A typical example is the integration of an optical repeater GaAs-GaAlAs laser with a MESFET reproduced here in Fig. 4. This phase of our work in described in some detail in [16], [38].

\section{B. The DFB Laser}

In 1972, a paper entitled "Coupled wave theory of distributed feedback lasers" by H. Kogelnik and C. V. Shank [17] proposed that the usual mirror feedback of a laser oscillator can be replaced by a continuous Bragg reflection provided by a spatially periodic modulation of the index or gain (loss) of a laser. This required that the spatial period of the modulation $\Lambda$ be equal to some integral multiple of half the guide wavelength

$$
\Lambda=m \frac{\lambda_{g}}{2} \quad m=1,2, \cdots .
$$

A number of experiments at various laboratories followed and demonstrated the distributed feedback (DFB) laser effect 


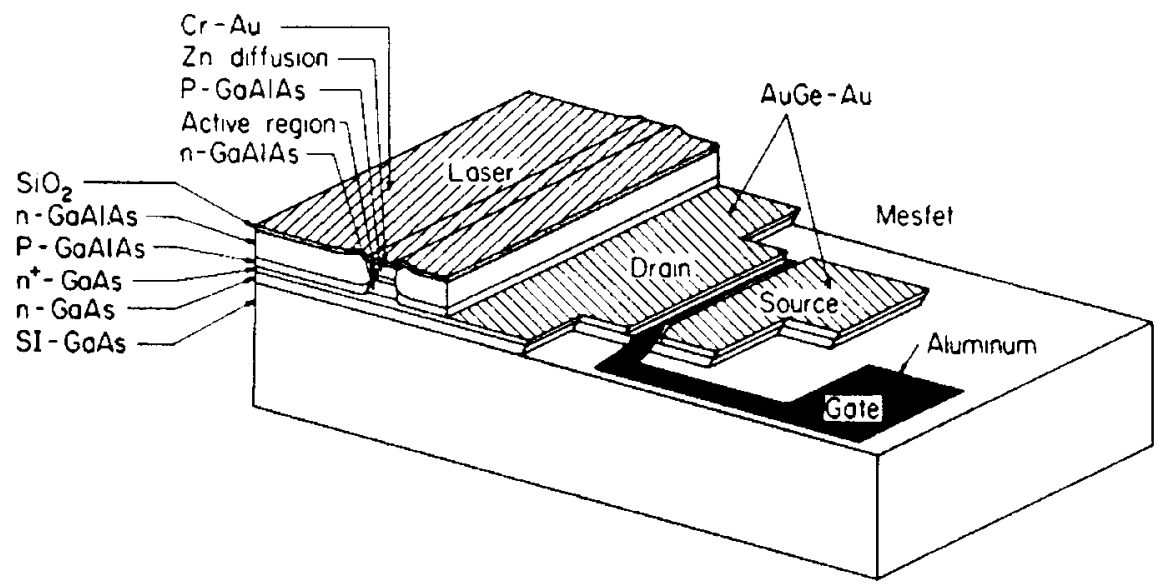

Fig. 4. A monolithic integration of a buried heterostructure GaAs-AlGaAs laser with a MESFET.

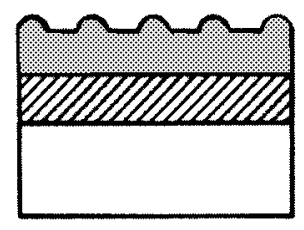

(a)
GaAs epitaxial layer $\mathrm{Ga}_{0.7} \mathrm{Al}_{0.3}$ As epitaxial layer GaAs substrate

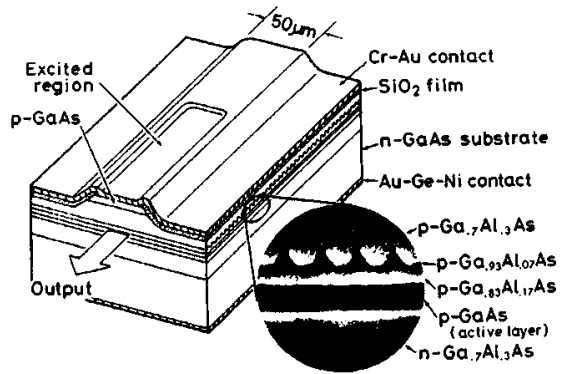

(b)

Fig. 5. (a) The optically pumped GaAs-GaAlAs corrugated DFB laser (1973). (b) A late mode electrically pumped GaAs-GaAlAs DFB laser.

by using a standing-wave optical pumping of dye lasers to obtain the requisite spatial modulation.

Probably the most dramatic aspect of this new type of laser was that the frequency of oscillation, in order to satisfy (8), was determined by the spatial period $\Lambda$. The Kogelnik-Shank paper hit us at the right time. We were already interested in periodic optical structures and K. Sakuda, a Japanese post-doc in my group, was studying propagation in periodic waveguides motivated by the possibility of making optical traveling wave amplifiers. We decided to bring to bear our newly acquired experience with GaAs laser crystal growth and try to make a DFB semiconductor laser. The task fell to H. W. Yen, a starting graduate student, M. Nakamura, a visitor from Hitachi's research laboratory, and Elsa Garmire. The key decision was to provide the requisite periodic modulation by a physical corrugation of one of the interfaces of the optical waveguide. A fundamental issue which troubled us at first was whether a surface perturbation which is localized transversely and thus only sensed by part of the mode field will not cause mostly scattering and little of the desired backward reflection (feedback). With the help of the coupled-mode theory [18], which I had just developed to treat interaction between modes in waveguides, it was clear that a truly periodic surface corrugation, by virtue of photon and "crystal" momentum conservation, can be made to couple only forward and backward traveling modes with essentially no scattering or losses to radiation modes.

In the first demonstration of a SC DFB laser [Fig. 5(a)], we used an optically pumped GaAs slab with a surface corruga- tion which (corrugation) was fabricated by a holographic-lithographic techniques [19]. The experiment is depicted in Fig. 5(b). This was followed in short order with an electrically pumped DFB laser with a "buried" active layer of GaAs sandwiched between layers of $\mathrm{Ga}_{1-x} \mathrm{Al}_{x} \mathrm{As}$. The corrugation was at the interface between the two crystals. Fig. 4 shows a third-generation laser, which is not too different from present-day commercial lasers. As anticipated, the DFB lasers oscillated in a single mode which was determined by the Bragg condition.

At that time, there was no particular need for a single-mode SCL, since the communication data rate was still in the megahertz range. The result was that most of the effort in DFB lasers died down by 1980 or so. The exception was the Hitachi Corporation. M. Nakamura returned to the Hitachi laboratories from Caltech and started a significant effort in DFB lasers. As the data rate of the then young optical fiber communication field kept increasing, the need for a SC laser emitting a single wavelength became acute. The multimode optical pulses of the Fabry-Perot lasers used at the time spread with distance, due to the fact that each mode propagates with a different group velocity. This severely limited the maximum data rate for a given communication distance. The DFB laser was, and still is, the only SC laser capable practically of providing the spectrally narrow light. For a number of years, Hitachi Corporation was the only company capable of supplying DFB lasers till eventually numerous other U.S. and non-U.S. companies started producing it. On a more personal basis, M. Nakamura was able to convert his Caltech work into a Ph.D. degree from Tokyo University. 


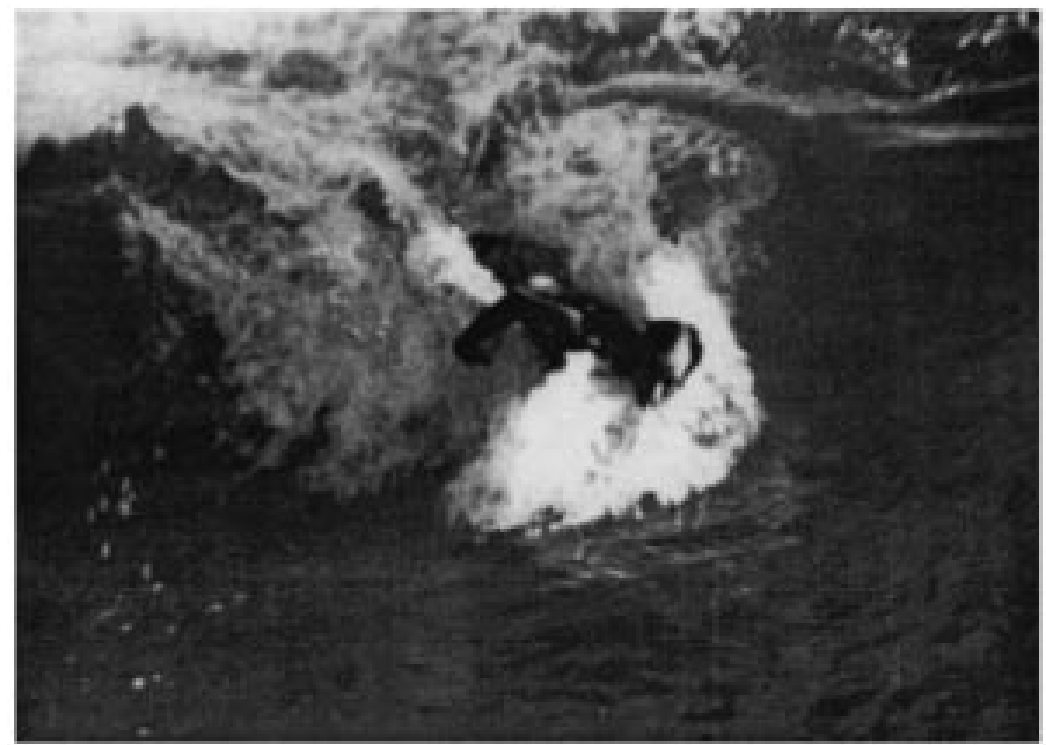

Fig. 6. "Catching" an $\mathrm{H}_{2} \mathrm{O}$ wave in Sandy Beach, 1980.

In 1980, Israel Ury, who had just obtained his Ph.D. degree from Caltech after demonstrating the first OEIC, and Nadav Bar-Chaim, a post-doctoral fellow in our group from Israel, and I founded ORTEL (Hill of Light in Hebrew) Corporation in Alhambra, CA. The company became a specialist in analog high-speed laser modulation and transmission and introduced it to cable TV transmission. In April 2000, the company was acquired by Lucent Corporation whose Bell Laboratories was my first employer some thirty years earlier.

On the personal side, my marriage to Jeanne did not survive the transition to California and, possibly, the work schedule of a young professor. In 1972, I was married to Frances and a couple of years later were joined by our daughter Gabriela. I did find some time to catch real waves, and in Fig. 6 you can see me riding an 8 -footer at Sandy Beach, Ohau, Hawaii, in 1980. This photo was rejected by a surfing magazine but I never gave up on publishing it.

\section{Phase Conjugation Optics}

At Caltech, in 1968 I started teaching a course, Applied Physics 190, on the physics of lasers and nonlinear optics. I would come back to teach the course after a hiatus of two or three years. Due to the rapid pace of development in the field, every time I would teach the course at least half the material would be new. In retrospect, there is something about teaching which I did not appreciate at first. The teaching of a course-especially to bright advanced students-forces the instructor to understand the material at a level which is much higher than that which he would achieve as, say, a worker in the field studying a topic for his own use. This is so because a professor cannot afford, too often, to appear ignorant to his class. I found, in my case, that the resulting heightened level of understanding was crucial for invention. This was an unexpected side benefit of teaching.

One example of this symbiotic relationship between teaching and research was our work on phase-conjugate optics. There were probably three or four independent strands of research worldwide that eventually converged to form the field of phaseconjugate optics. I will tell here the Caltech story. It started in my Aph 190 class after we derived the propagation modes $E_{m n}(x, y) e^{-i \beta_{m n} z}$ of modes in a quadratic index (QI) fiber. To illustrate the significance of the propagation constant $\beta_{m n}$, I considered the case of transmitting a "picture" $p(x, y)$ which is imaged on the front facet, $z=0$, of a multimode thick QI fiber. The picture excites a superposition of the modes of the fiber $E_{m n}(x, y)$

$$
p(x, y)=\sum_{m, n} a_{m n} E_{m n}(x, y)+\text { c.c. }
$$

so that the pictorial information resides in the $a_{m n}$ coefficients. The input field will then propagate to an arbitrary plane $z=L$ as

$$
p(x, y, L)=\sum_{m, n} a_{m n} E_{m n}(x, y) e^{-i \beta_{m n} L}+\text { c.c. }
$$

where $\beta_{m n}$ of the propagation constant of mode $m n$ is obtained by solving the propagation equation. It is clear that in the general case $p(x, y, L) \neq p(x, y)$ due to the accumulated extra phases $-\beta_{m n} L$. The original picture is thus blurred in transmission. But the blurring due to the extra phase $-\beta_{m n} L$ acquired by each mode $(m, n)$ is wholly deterministic. From the information theory point of view, the pictorial information was not lost or degraded but merely decoded into a new manifold. To recover the original picture, there exist two routes. 1) add to each mode a phase $+\beta_{m n} L$ to cancel out the $-\beta_{m n} L$ "bad" phase so that the new field $p^{\prime}$ is identical to the input field $p(x, y)$

$$
\begin{aligned}
p^{\prime}(x, y, L) & =\sum_{m, n} a_{m n} E_{m n}(x, y) e^{-i\left(\beta_{m n}-\beta_{m n}\right) L}+\text { c.c. } \\
& =p(x, y) .
\end{aligned}
$$

Although this solution is mathematically perfect, we could not then, nor now, think of a way to tag a unique phase $\beta_{m n} L$ on each mode $(m, n)$. 2) A second approach that at first hand seemed even more convoluted was to change at $z=L$, by some 
means the phase $\left(-\beta_{m n} L\right)$ of each mode to its inverse $+\beta_{m n} L$, resulting in

$$
p^{\prime \prime}(x, y, L)=\sum_{m, n} a_{m n} E_{m n}(x, y) e^{i \beta_{m n} L}+\text { c.c. }
$$

and then allow $p^{\prime \prime}(x, y, L)$ to propagate through a second leg in the same fiber of the same length $L$ so that at $z=2 L$

$$
\begin{aligned}
p^{\prime \prime \prime}(x, y, 2 L) & =\sum_{m, n} a_{m n} E_{m n}(x, y) e^{-i\left(\beta_{m n}-\beta_{m n}\right) L}+\text { c.c. } \\
& =p(x, y)
\end{aligned}
$$

and the original picture would be restored. The advantage of this method was that we already knew, but were not aware of it, how to change the phase of a coherent electromagnetic field from $\theta$ to $-\theta$. The solution to this problem I realized, one day, lay in nonlinear optics [20]. In this field, we were mostly focused up to that time on the possibility of "adding" or "subtracting" optical frequencies. As an example-if we "multiply" in some nonlinear crystal a field at $2 \omega$ with a field at $\omega$, we can generate a new field at $\omega$ due to the nonlinear polarization term involving the product of the two

$$
\left[E^{2 \omega} e^{i 2 \omega t}+\left(E^{2 \omega}\right)^{*} e^{-i 2 \omega t}\right]\left[E^{\omega} e^{i \omega t}+\left(E^{\omega}\right)^{*} e^{-i \omega t}\right]
$$

which is proportional to $E^{2 \omega}\left(E^{\omega}\right)^{*} e^{i \omega t}$. In other words, the complex field amplitude $E^{\omega}$ was transformed to one proportional to $\left(E^{\omega}\right)^{*}$ so that the phase of $E^{\omega}$ changed sign. If we replace $E^{\omega}$ with the picture field $p(x, y, L)$ of (10) the result of the nonlinear interaction in the crystal of $p(x, y, L)$ with the field $E^{2 \omega} e^{i 2 \omega t}$ was the field

$$
\begin{aligned}
p^{\prime \prime}(x, y, L) & \propto E^{2 \omega} e^{i 2 \omega t} \sum_{m, n} a_{m n}^{*} E_{m n}^{*}(x, y) e^{i \beta_{m n} L} e^{-i \omega t}+\text { c.c. } \\
& =E^{2 \omega} \sum_{m n} a_{m n}^{*} E_{m n}^{*} e^{i\left(\beta_{m n} L+\omega t\right)}+\text { c.c. }
\end{aligned}
$$

i.e., just the desired field of (12). (I included the time factor to show that the original frequency is recovered.) This field then propagates to $z=2 L$ and becomes

$$
p^{\prime \prime}(x, y, 2 L) \propto E^{2 \omega} e^{i \omega t} \sum_{m, n} a_{m n}^{*} E_{m n}^{*}(x, y)+\text { c.c. }
$$

The complex amplitude of this field $E^{2 \omega} \sum_{m, n}$ $a_{m n}^{*} E_{m, n}^{*}(x, y)$ is the complex conjugate of the original picture field $p(x, y)$, thus corresponding to the same intensity (pictorial) distribution.

Although, in principle, our solution was realizable, the need for an additional field at $2 \omega$ of sufficient intensity and the need for phase matching in the phase conjugation process made the whole setup exceedingly difficult. Its main contribution was to point the way to nonlinear optics as a method to obtain the complex conjugate of an optical field. The practical way of doing what came to be known as "Phase Conjugation Optics" is due to W. Hellwarth of USC, a friend and, at that time, a fellow consultant with me at the Hughes Research Laboratories in Malibu, where we mostly consulted with each other on phase conjugate optics. His solution was to use four-wave mixing [21] (i.e.,
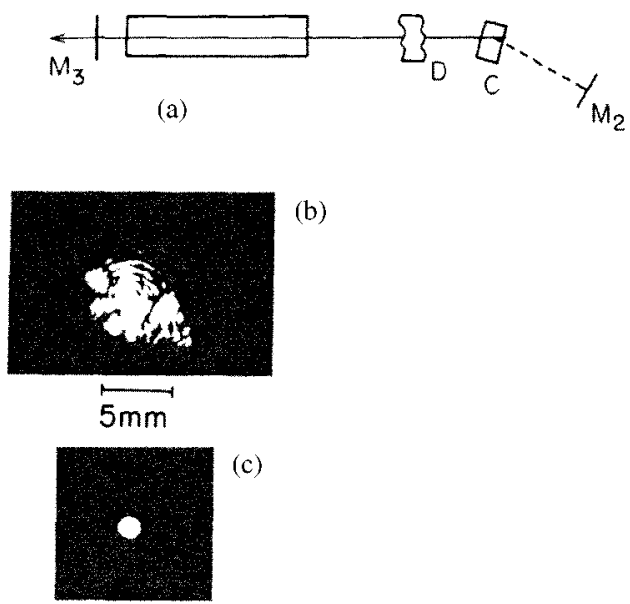

(b)

Fig. 7. A phase conjugate reflector used as a laser resonator mirror compensates in real time for a distortion $D$ placed within the resonator. (a) The experimental setup. (b) The laser output with the distorter when two conventional reflectors form the resonator. (c) The output beam with one of the reflectors replaced by a PC mirror. From [26].

third-order optical nonlinearity) so that, instead of (14), one would get

$$
\begin{aligned}
p^{\prime \prime}(x, y, L) & \propto E^{\omega} e^{i \omega t} E^{\omega} e^{i \omega t} \sum_{m n} a_{m n}^{*} E_{m n}^{*}(x, y) e^{-i \omega t} \\
& =\left(E^{\omega}\right)^{2} e^{i \omega t} \sum_{m n} a_{m n}^{*} E_{m n}^{*}(x, y)
\end{aligned}
$$

so that only fields at $\omega$ are involved.

Although nobody has seriously attempted using phase conjugation for pictorial transmission, a slight variant of the basic idea is one of the serious candidates for compensating pulse, spreading due to group velocity dispersion in high data rate transmission in modern day optical fibers [22], [23]. Another interesting aspect of our work on phase-conjugate optics served to illustrate again the power of the coupled-mode formalism. When we applied it to the case of phase conjugation by four-wave mixing, the solution for the reflected and conjugated wave predicted that its magnitude could exceed that of the input wave. In other words, the phase-conjugating medium could also act as an amplifier [24]. This possibility was not foreseen by any of us, or any of our colleagues, and "dropped" on us as a mathematical consequence. ${ }^{1}$ The phase conjugation gain can become infinite so that the device could oscillate [25]. Fig. 7 demonstrates dramatically the "magic" of phase-conjugate optics and its real-time holographic nature. The phase-conjugate reflector acting as an end mirror in a laser oscillator [26] compensates essentially perfectly for the presence of a severe distortion (D) inside the resonator.

\section{Periodic Structures, Photonic Crystals, and Defect Mode Cavity Lasers}

My interest in periodic structures has been probably the single most constant element of my scientific pursuits. It started with the periodic microwave guides of the traveling-wave

\footnotetext{
${ }^{1}$ It has now become part of our physical intuition which comes to show that physical intuition is often just mathematical consequences with which we have lived long enough to make them part of our "world picture."
} 


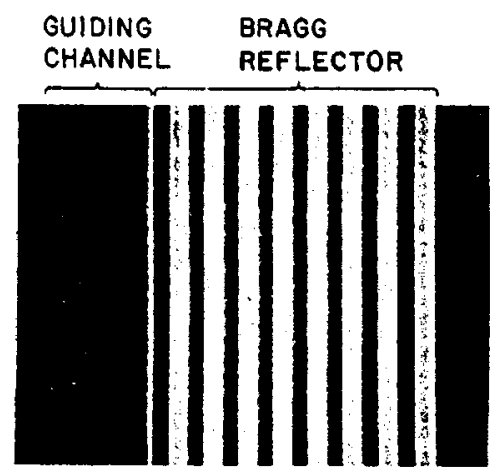

$1 \mu m$

(a)

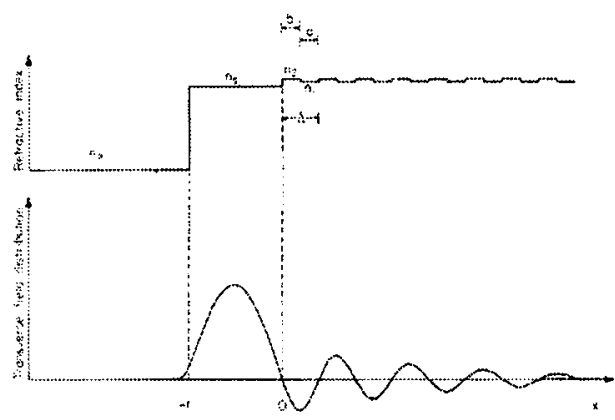

(b)

Fig. 8. Guiding in a Bragg waveguide made up of a periodic stack of GaAs-GaAlAs layers. (a) The structure. (b) The measured modal intensity profile.

tubes during my Berkeley days and continued with our work at Caltech on one-dimensional periodic optical waveguides and the closely related DFB semiconductor laser. Around 1976, we realized that Bragg reflection could also be used to create a new type of optical waveguide. A wave incident on a periodic stack at the Bragg angle, or near it, can be reflected nearly perfectly from only a small number of unit cells (layers), provided the index difference between layers is sufficiently large. This happens while the field penetrating into the periodic stack evanesces exponentially. A medium, say air, sandwiched between two such stacks forms a waveguide, since a ray in the air layer can zigzag by repeated Bragg reflections down the layer. Pochi Yeh as a student in the group (Pochi is now a professor at the University of California at Santa Barbara) wrote an elegant thesis on light propagation in periodic media and, in an experiment on samples grown by $\mathrm{Al}$ Cho of Bell labs, demonstrated optical wave guiding in a Bragg waveguide [27], [39]. Fig. 8 shows such a guide in GaAs.

In the 1980's and early 1990's, our semiconductor laser work was concentrated on noise and dynamic aspects. Kerry Vahala, now a professor at Caltech, in his doctoral research was able to incorporate the phase amplitude coupling mechanism suggested by M. Lax [28] and later by C. H. Henry [29] into a first principles laser theory [30] which explained successfully the observed linewidth and spectral properties of these lasers. An important consequence of this theory was the prediction and observation of two satellite noise peaks in the laser spectrum separated from the main peak by the relaxation resonance frequency. This model was also important in elucidating the conversion of $\mathrm{FM} \leftrightarrow \mathrm{AM}$ noise in propagation of SCL light in optical communication fibers. A two year visit (1982-1984) by Dr. Yasuhiku Arakawa of Tokyo University made us reorient much of our subsequent SC laser research toward quantum well and other quantum-confined structures. Arakawa, who had started this work in Japan, was able to convince us that these new structures were superior to conventional lasers [31].

Our phase-conjugate optics work during this period concentrated on issues related to holographic data storage in photorefractive crystals. These holograms are essentially periodic index patterns in the crystal ("frozen" electric charge waves) operating in the Bragg regime so that, in a way, we were still dealing with

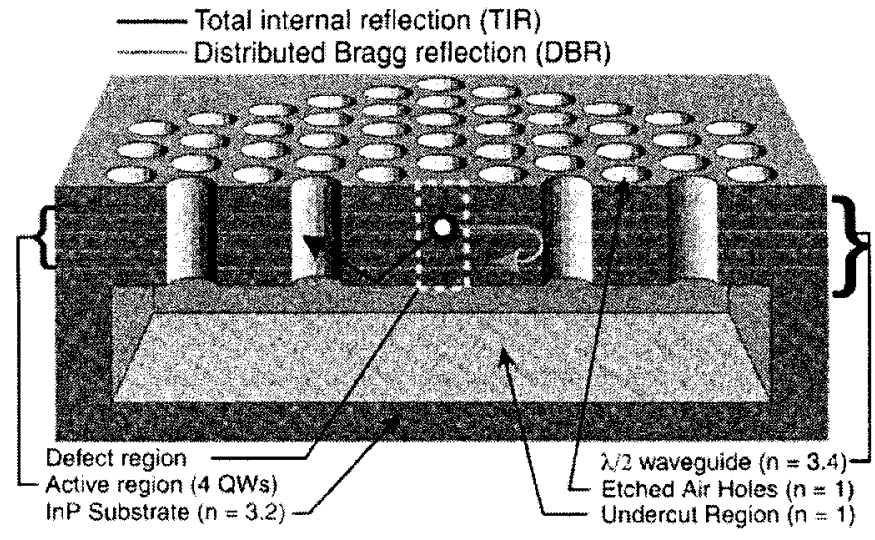

Fig. 9. A periodic $(2+1)$ D structure used to fabricate a defect mode laser and microcavities.

periodic structures. The main legacy of our work during this period is the suggestion and analysis of holographic memories based on wavelength multiplexing and the use of reciprocal momentum $\vec{K}$ in analyzing multihologram storage [32].

In 1993, we were fortunate and succeeded in attracting to Caltech Axel Scherer of Bellcore, one of the world's leading experts in nanofabrication and material processing. Axel had been trying with some limited success to produce by etching and drilling three-dimensional (3-D) periodic structures- - "photonic crystals" - which have been proposed by Eli Yablonovitch [33]. These 3-D structures were hellishly difficult to fabricate and were possibly of limited usefulness because of the difficulty of optical and electrical connection to them. Consequently, Axel and I decided to shift our emphasis to a new class of periodic structures and combined our efforts and our students to this goal. These consisted of a two-dimensional (2-D) conventional optical waveguide which provides vertical confinement of light by total internal reflection. Horizontal confinement of light is then provided by a periodic array of holes drilled normal to the plane of the waveguide. An example of such structure is shown in Fig. 8. Unlike true 3-D photonic crystals, some light can still escape from this new geometry in thevertical direction due to rays incident on the interfaces at angles exceeding that of total internal reflection. To underscore this difference, we called this 


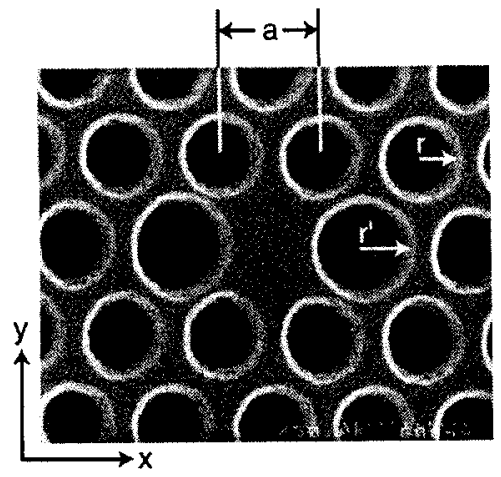

(a)

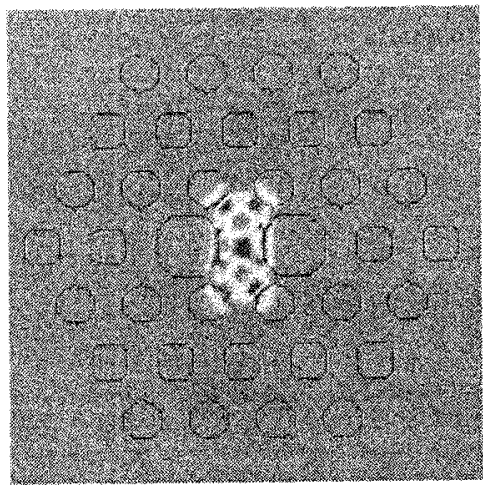

(b)

Fig. 10. A defect mode cavity in a $(2+1)$ D structure. (a) The "defect" (top view, for a cutaway, see Fig. 9). (b) The calculated field distribution.

form of wave confinement not 3 (dimensional) but $2+1$ (where $2+1 \neq 3)$.

An excellent group of graduate students from both our research groups, which included Brian D’Urso, Yong Xu, Reginald Lee, and Oscar Painter, developed the complicated numerical programs for solving Maxwell's equation in these structures. These structures formed the basis for a new type of an optical cavity, illustrated in Fig. 9. To see how this works, consider again Fig. 9. If in a perfectly periodic $2+1$ structure we leave out one hole, i.e., we "plug" it up, light generated inside this "defect" is confined by waveguiding in the $z$ direction, normal to the plane. It is confined in the lateral $x, y$ directions by total internal reflection from the periodic array of holes provided its frequency is within the Bragg ("forbidden") region. The "defect" thus constitutes an optical resonator since light is confined within its volume, penetrating only evanescently into the surrounding periodic medium. These resonators have a major advantage over true 3-D resonators, as yet not demonstrated, in that they can be contacted electrically or optically via closely coupled waveguides. Also, a series of such defects in close proximity can form a new type of waveguide with dispersion properties radically different from those of conventional waveguides [34]. Reginald Lee and Oscar Painter dedicated the bulk of their $\mathrm{Ph}$.D. research to demonstrating laser action in such defect resonators. The structure used to realize laser action in this new type of resonator utilized GaInAsP laser wafers provided by Dan Dapkus of USC and Jeff Ungar at ORTEL Corporation and were pumped optically. Fig. 8 shows a defect laser in this material [35]. The laser demonstrated in the figure has a volume smaller than $\left(\lambda_{0} / 2 n\right)^{3}$ and is the smallest laser demonstrated to date. Since in this small volume only one or two resonator modes fall within the gain (spectral region) of the pumped semiconductor material, the spontaneous emission lifetime, the noise, and dynamic properties are expected to differ radically from those of "conventional" SC lasers. We will probably devote the next few years to exploring these properties as well as investigate means for electrical and optical access to these structures. I believe that the next major development in the control and manipulation of light and harnessing it to computation and communication purposes will come from the domain of microresonators and lasers.

Looking back, I am surprised by the wonderful and exciting opportunities afforded me by the study of waves. Particularly exciting was, and still is, the opportunity to surf these waves together with the very talented graduate students at the unique setting and ambience of my (scientific) home for 36 years-Caltech.

\section{REFERENCES}

[1] J. R. Pierce, "Coupling of modes of propagation," Appl. Phys., vol. 25, pp. 179-183, 1954

[2] A. Yariv, "On the coupling coefficients in the coupled mode theory," Proc. IRE, vol. 46, no. 12, 1954.

[3] J. A. Armstrong, N. Bloembergen, J. Ducuing, and P. S. Pershan, "Interaction between light waves in nonlinear dielectric," Phys. Rev., vol. 127, p. $1918,1962$.

[4] N. Bloembergen and R. V. Pound, Phys. Rev., vol. 95, p. 8, 1954.

[5] K. Shimoda, H. Takahashi, and C. H. Townes, J. Phys. Soc. Japan, vol. 12, p. 686, 1957.

[6] W. H. Louisell, A. Yariv, and A. E. Siegman, "Quantum fluctuations and noise in parametric processes," Phys. Rev., vol. 124, pp. 1646-1654, 1961.

[7] L. Wu, H. J. Kimble, J. L. Hall, and H. Wu, "Generation of squeezed state by parametric down conversion," Phys. Rev. Lett., vol. 57, p. 2520, 1986.

[8] H. Kogelnik and A. Yariv, "Considerations of noise and schemes for its reduction in laser amplifiers," Proc. IEEE, vol. 52, 1964.

[9] A. Yariv and R. C. C. Leite, "Dielectric waveguide mode of light propagation in p-n junctions," Appl. Phys. Lett., vol. 2, pp. 55-77, 1962.

[10] K. Gürs and R. Müller, Phys. Lett., vol. 5, p. 179, 1963.

[11] A. Yariv, "Internal modulation in multimode laser oscillators," J. Appl. Phys., vol. 36, pp. 388-391, 1965.

[12] L. E. Hargrove, R. L. Fork, and M. A. Pollack, Appl. Phys. Lett., vol. 5, no. 4, 1964.

[13] D. Hall, A. Yariv, and E. Garmire, "Observation of propagation cutoff and its control in thin optical waveguides," Appl. Phys. Lett, vol. 17, pp. $127-129,1970$.

[14] R. Shubert and J. H. Harris, "Optical surface waves on thin films and their application to integrated data processors," IEEE Trans. Microwave Theory Tech., vol. 16, p. 1048, 1968.

[15] A. Yariv, "Active integrated optics," in Proc. Esfahan Conf. Fundamental and Applied Laser Physics, 1973, p. 897.

[16] _ _ "The beginning of integrated optoelectronic circuits," IEEE Trans. Electron. Devices, vol. ED-31, pp. 1656-1661, Nov. 1984.

[17] H. Kogelnik and C. V. Shank, "Coupled wave theory of distributed feedback lasers," J. Appl. Phys., vol. 43, p. 2328, 1972.

[18] A. Yariv, "Coupled-mode theory for guided-wave optics," IEEE $J$. Quantum Electron., vol. QE-9, pp. 919-933, 1973.

[19] H. W. Yen, M. Nakamura, E. Garmire, S. Somekh, A. Yariv, and H. L. Garvin, "Optically pumped GaAs waveguide lasers with a fundamental $0.11 \mu$ corrugated feedback," Opt. Commun., vol. 9, pp. 35-37, 1973.

[20] A. Yariv, "Compensation for atmospheric degradation of optical beam transmission by nonlinear optical mixing," Opt. Commun., vol. 21, pp. 49-50, 1977.

[21] R. Hellwarth, J. Opt. Soc. Amer, vol. 67, p. 1, 1977. 
[22] A. Yariv, D. Fekete, and D. M. Pepper, "Compensation for channel dispersion by nonlinear optical phase conjugation," Opt. Lett, vol. 4, pp. 52-54, 1979.

[23] S. Watanabe, T. Naito, and T. Chikama, "Compensation of chromatic dispersion in a single mode fiber by optical phase conjugation," IEEE Photon. Technol. Lett., vol. 5, p. 92, 1993.

[24] A. Yariv and D. Pepper, "Amplified reflection, phase conjugation and oscillation in degenerate four-wave mixing," Opt. Lett., vol. 1, p. 16, 1977.

[25] D. Pepper, D. Fekete, and A. Yariv, "Observation of amplified phaseconjugation reflection and optical parametric oscillation by degenerate four-wave mixing in a transparent medium," Appl. Phys. Lett., vol. 33, pp. 41-44, 1978.

[26] M. Cronin-Golomb, B. Fischer, J. Nilsen, J. O. White, and A. Yariv, “A laser with dynamic holographic intracavity distortion correction capability," Appl. Phys. Lett., vol. 41, pp. 219-220, 1982.

[27] A. Y. Cho, A. Yariv, and P. Yeh, "Observation of confined propagation in Bragg waveguides," Appl. Phys. Lett., vol. 30, p. 471, 1977.

[28] M. Lax, "Classical noise vs. noise in self sustained oscillators," Phys. Rev., vol. 160, pp. 290-307, 1967.

[29] C. H. Henry, "Theory of linewidth in semiconductor lasers," IEEE J. Quantum Electron., vol. QE-18, pp. 259-264, 1982.

[30] K. Vahala and A. Yariv, "Semiclassical theory of laser noise-Part I, II," IEEE J. Quantum Electron., vol. QE-19, pp. 1096-1109, 1983.

[31] Y. Arakawa and A. Yariv, "Theory of gain, modulation response, and spectral linewidth in AlGaAs quantum confined lasers," IEEE $J$. Quantum Electron., vol. QE-21, p. 1966, 1985.
[32] G. Rakuljic, V. Leyva, and A. Yariv, "Optical data storage by using orthogonal wavelength-multiplexed volume holograms," Opt. Lett., vol. 17, pp. 1471-1473, 1992.

[33] E. Yablanovitch, Phys. Rev. Lett., vol. 58, p. 2059, 1987.

[34] A. Yariv, Y. Xu, R. K. Lee, and A. Scherer, "Coupled-resonator optical waveguide: A proposal and analysis," Opt. Lett., vol. 24, pp. 711-713, 1999.

[35] O. Painter, R. K. Lee, A. Scherer, A. Yariv, J. D. Obrien, D. D. Dapkus, and I. Kim, "Two dimensional photonic band-gap defect mode laser," Science, vol. 284, p. 1819, 1999.

[36] E. M. Purcell and R. V. Pound, Phys. Rev., vol. 81, p. 279, 1951.

[37] W. Bond, B. G. Cohen, R. C. C. Leite, and A. Yariv, "Observation of the dielectric waveguide mode in p-n junction," Appl. Phys. Lett., vol. 2, p. 57, 1963.

[38] M. Yust et al., "A monolithically integrated optical repeater," Appl. Phys. Lett., vol. 10, p. 795, 1979.

[39] P. Yeh, A. Yariv, and C. S. Heng, "Electromagnetic propagation in periodic stratified media I: General theory," J. Opt. Soc. Amer., vol. 67, pp. 423-438, 1977.

Amnon Yariv (S'56-M'59-F'70-LF'95), photograph and biography not available at the time of publication. 